\title{
Article \\ On the Probabilistic Deployment of Smart Grid Networks in TV White Space
}

\author{
Angela Sara Cacciapuoti ${ }^{1,2, *}$, Marcello Caleffi ${ }^{1,2}$ and Luigi Paura ${ }^{1,2}$ \\ 1 Department of Electrical Engineering and Information Technologies, University of Naples Federico II, \\ Naples 80138, Italy; marcello.caleffi@unina.it (M.C.); luigi.paura@unina.it (L.P.) \\ 2 Multimedia Communications Laboratory, CNIT, Naples 80126, Italy \\ * Correspondence: angelasara.cacciapuoti@unina.it; Tel.: +39-081-768-3793
}

Academic Editor: Leonhard M. Reindl

Received: 29 February 2016; Accepted: 6 May 2016; Published: 10 May 2016

\begin{abstract}
To accommodate the rapidly increasing demand for wireless broadband communications in Smart Grid (SG) networks, research efforts are currently ongoing to enable the SG networks to utilize the TV spectrum according to the Cognitive Radio paradigm. To this aim, in this letter, we develop an analytical framework for the optimal deployment of multiple closely-located SG Neighborhood Area Networks (NANs) concurrently using the same TV spectrum. The objective is to derive the optimal values for both the number of NANs and their coverage. More specifically, regarding the number of NANs, we derive the optimal closed-form expression, i.e., the closed-form expression that assures the deployment of the maximum number of NANs in the considered region satisfying a given collision constraint on the transmissions of the NANs. Regarding the NAN coverage, we derive the optimal closed-form expression, i.e., the closed-form expression of the NAN transmission range that assures the maximum coverage of each NAN in the considered region satisfying the given collision constraint. All the theoretical results are derived by adopting a stochastic approach. Finally, numerical results validate the theoretical analysis.
\end{abstract}

Keywords: white space; smart grid; cognitive radio; sensor network

\section{Introduction}

To accommodate the rapidly increasing demand for wireless broadband communications in Smart Grid (SG) scenarios, research efforts [1] are currently ongoing to enable the SG Networks (SGNs) to utilize the channels of the TV spectrum temporarily unused by the licensed users, referred to as incumbents, according to the Cognitive Radio paradigm [2-6]. The vacant channels are known as TV White Space (TVWS) channels.

SG communications over TVWS channels are conditioned by regulators on the ability of the SGNs to avoid harmful interference toward incumbents. To this aim, the existing rulings [7] obviate the spectrum sensing as the mechanism for the SGNs to determine the TVWS availability. Instead, they require the SGNs to periodically access to a geolocated database, referred to as White Space DataBase (WSDB) [8-10], for acquiring the list of TVWS channels.

Let us consider the typical SG scenario shown in Figure 1, where several smart meters connected to a gateway constitute a Home Area Network (HAN), and multiple gateways connected to a Data Aggregate Unit (DAU) constitute a Neighborhood Area Network (NAN) $[1,11,12]$ (please note that the typical wireless technologies and the corresponding values of the coverage ranges for HANs and NANs are reported in [12], along with further details on their communication features). The gateway is responsible for transmitting the meter data periodically collected within its HAN to the DAU via a TVWS channel, once declared available by the WSDB. However, experimental studies have shown that the number of TVWS channels is significantly limited in urban areas [13]. Hence, it is likely that 
multiple closely-located NANs are authorized to use the same TVWS channel and their transmissions may collide, leading so to a performance degradation. Moreover, since so far there are no regulatory requirements for the coexistence among NANs operating in TVWS spectrum, such a performance degradation can be severe. The described scenario of multiple closely-located NANs sharing the same TVWS channel has been considered for the first time in [14], where the sensing duration that maximizes the achievable data rate at a given NAN has been determined.

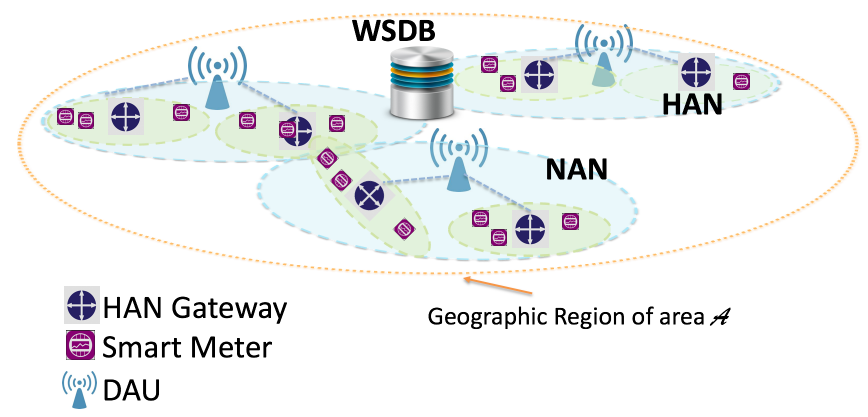

Figure 1. Smart Grid Scenario: Multiple Closely-Located Neighborhood Area Networks (NANs).

Differently from [14], in this letter, we develop an analytical framework for the optimal deployment of multiple closely-located NANs using the same TVWS channel with the objective to derive the optimal values for both the number of NANs and their coverage. More specifically, regarding the number of NANs, we derive the optimal closed-form expression, i.e., the closed-form expression that allows to evaluate the maximum number of NANs in the considered network region satisfying a given collision constraint on the transmissions of NANs.

Regarding the NAN coverage, we derive the optimal closed-form expression, i.e., the closed-form expression of the NAN transmission range that assures the maximum coverage of each NAN in the considered network region satisfying the given collision constraint.

All theoretical results are derived by adopting a stochastic approach and by considering the specificity of the NAN traffic demands. Finally, numerical results validate the theoretical analysis.

\section{System Model}

In a geographic region of area $\mathcal{A}, K$ NANs are deployed. The HAN gateways of each NAN are responsible for transmitting the meter data periodically collected within their HANs to the DAU via the same TVWS channel declared available by the WSDB, as depicted in Figure 1. We denote with $G_{i}$ the number of HAN gateways belonging to the $i$-th NAN and with $R_{\mathrm{TX}}^{i}$ their transmission range. The traffic of the $i$-th NAN is modeled thought the parameters $P_{\mathrm{a}}^{i}$ and $P_{\mathrm{in}}^{i} \triangleq 1-P_{\mathrm{a}}^{i}$ denoting the probabilities of an arbitrary HAN gateway belonging to the $i$-th NAN being active (i.e., having meter data collected within its HAN to transmit to the DAU) and inactive, respectively.

\section{Optimal Deployment Analysis}

At a certain time instant $t$, the transmissions of two arbitrary HAN gateways, say $H_{\ell}^{i}$ and $H_{m}^{j}$ belonging to the $i$-th and $j$-th NANs, respectively, collide if and only if the HAN gateways are both active and their distance $d_{H_{\ell}^{i} H_{m}^{j}}(t)$ (denoted in the following as $d_{i j}(t)$ for the sake of simplicity) is smaller than the distance threshold constituted by the maximum between their transmission ranges, i.e., $d_{i j}(t) \leq \max \left(R_{\mathrm{TX}}^{i}, R_{\mathrm{TX}}^{j}\right)$. Hence, it results that the transmissions of a certain HAN gateway belonging to the $i$-th NAN, say $H_{\ell}^{i}$, collide with the transmissions of the $j$-th NAN if it exists al least one active HAN gateway belonging to the $j$-th NAN within the distance threshold. We denote the probability of this event as $P_{\mathrm{C}}^{H_{\ell}^{i}, j}$. As a consequence, there is no collision between the transmissions of 
NANs $i$ and $j$ if none of the active HANs belonging to NAN $i$ is within the distance threshold of any of the active HANs belonging to NAN $j$. We denote the probability of this event as $\bar{P}_{\mathrm{C}}^{i j}$. In the following, by accounting for these definitions, with some algebraic manipulations, we derive the expressions of $P_{\mathrm{C}}^{H_{\ell}^{i}, j}$ and $P_{\mathrm{C}}^{i j} \triangleq 1-\bar{P}_{\mathrm{C}}^{i j}$. Specifically:

$$
P_{\mathrm{C}}^{H_{\ell^{\prime}}^{i}} \triangleq 1-\bar{P}_{\mathrm{C}}^{H_{\ell, j}^{i}}=1-\left[P_{\mathrm{in}}^{i}+P_{\mathrm{a}}^{i}\left(1-P_{\mathrm{a}}^{j} P_{d_{i j}}\right)^{G_{j}}\right]
$$

where $P_{d_{i j}}$ denotes the probability of the event $\left\{d_{i j}(t) \leq \max \left(R_{\mathrm{TX}}^{i}, R_{\mathrm{TX}}^{j}\right)\right\}$. By accounting for Equation (1) and the definition of $P_{C}^{i j}$, in the reasonable hypothesis of independence of the HAN transmissions, it results

$$
P_{\mathrm{C}}^{i j} \triangleq 1-\bar{P}_{\mathrm{C}}^{i j}=1-\prod_{l=1}^{G_{i}} \bar{P}_{\mathrm{C}}^{H_{\ell}^{i}, j}=1-\left[P_{\text {in }}^{i}+P_{\mathrm{a}}^{i}\left(1-P_{\mathrm{a}}^{j} P_{d_{i j}}\right)^{G_{j}}\right]^{G_{i}}
$$

Since in a typical SG scenario it is reasonable to assume statistically the NANs having similar traffic and communication characteristics, the collision probabilities between two NANs are symmetric, i.e., $P_{\mathrm{C}}^{i j}=P_{\mathrm{C}}^{j i}=P_{\mathrm{C}}$. Hence the overall collision probability $P_{\mathrm{C}}^{\mathcal{O}}$, i.e., the probability that the transmissions of at least one NAN collide with the transmissions of at least one of the remaining $(K-1)$ NANs, can be calculated as follows:

$$
P_{\mathrm{C}}^{\mathcal{O}} \triangleq 1-\left(\bar{P}_{\mathrm{C}}\right)^{\left(\begin{array}{l}
K \\
2
\end{array}\right)}=1-\left(\bar{P}_{\mathrm{C}}\right)^{\frac{K(K-1)}{2}}
$$

where $\bar{P}_{C}=\bar{P}_{C}^{i j}$ is given in Equation (2) and the binomial coefficient $\left(\begin{array}{l}K \\ 2\end{array}\right)$ is due to the symmetry of the collision probabilities $\left\{P_{C}^{i j}\right\}_{i, j \in\{1, \ldots K\}}$. By substituting $\bar{P}_{\mathrm{C}}$ in Equation (3), one has:

$$
P_{\mathrm{C}}^{\mathcal{O}}=1-\left[P_{\text {in }}+P_{\mathrm{a}}\left(1-P_{\mathrm{a}} P_{d}\right)^{G}\right]^{\frac{G K(K-1)}{2}}
$$

As mentioned in the introduction, $P_{C}^{\mathcal{O}}$ has to be under a threshold, depending on the considered application, to assure a certain level of protection on the NANs transmissions, i.e.,

$$
P_{\mathrm{C}}^{\mathcal{O}} \leq \mathcal{T}_{\mathrm{C}}
$$

With $\mathcal{T}_{\mathrm{C}}$ denoting the largest value of the overall collision probability tolerated by the SG scenario. In the following we refer to $\mathcal{T}_{C}$ as collision constraint. Satisfying Equation (5) is equivalent to satisfy:

$$
1-\left[P_{\text {in }}+P_{\mathrm{a}}\left(1-P_{\mathrm{a}} P_{d}\right)^{G}\right]^{\frac{G K(K-1)}{2}} \leq \mathcal{T}_{\mathrm{C}}
$$

The largest value of $K$ satisfying Equation (6), say the optimal value $K_{\text {opt }}$, is the maximum number of NANs that can be deployed in the region of area $\mathcal{A}$ satisfying the collision constraint $\mathcal{T}_{\mathrm{C}}$. Specifically, by applying the logarithm function with basis $P_{\mathrm{in}}+P_{\mathrm{a}}\left(1-P_{\mathrm{a}} P_{d}\right)^{G}$ to Equation (6) and by recognizing that this logarithm is a decreasing function of its argument since $P_{\text {in }}+P_{\mathrm{a}}\left(1-P_{\mathrm{a}} P_{d}\right)^{G} \leq 1$, one has:

$$
P_{\mathrm{C}}^{\mathcal{O}} \leq \mathcal{T}_{\mathrm{C}} \Leftrightarrow K(K-1) \leq \frac{2}{G} \log _{P_{\mathrm{in}}+P_{\mathrm{a}}\left(1-P_{\mathrm{a}} P_{d}\right)^{G}}\left(1-\mathcal{T}_{\mathrm{C}}\right)
$$

Hence, $K_{o p t}$ is obtained as the largest integer less than or equal to the maximum value satisfying Equation (7):

$$
K_{\mathrm{opt}}=\left\lfloor\frac{1}{2}\left(-1+\sqrt{1+\frac{8}{G} \log _{P_{\mathrm{in}}+P_{\mathrm{a}}\left(1-P_{\mathrm{a}} P_{d}\right)^{G}}\left(1-\mathcal{T}_{\mathrm{C}}\right)}\right)\right\rfloor
$$


With $\lfloor\cdot\rfloor$ denoting the floor operator. It's easy to verify that $1+\frac{8}{G} \log _{P_{\text {in }}+P_{\mathrm{a}}\left(1-P_{\mathrm{a}} P_{d}\right)^{G}}\left(1-\mathcal{T}_{\mathrm{C}}\right) \geq 1$, i.e., $K_{\mathrm{opt}}$ is positive. From Equation (8) it results that, for a given value of $\mathcal{T}_{\mathrm{C}}, K_{\mathrm{opt}}$ decreases as $G$ increases. The same consideration holds if $P_{\mathrm{a}}$ increases.

In the hypothesis of NANs uniformly distributed in the region of area $\mathcal{A}$ and $R_{\mathrm{TX}}<<\sqrt{\mathcal{A}}$ (We assumed a bi-dimensional squared network region. This hypothesis is not restrictive. In fact, if the network region has dimensions $a$ and $b$, respectively, one has to compare the transmission range with the smallest dimension, i.e.: $\left.R_{T X}<<\min \{a, b\}\right), P_{d}=P\left(d(t)<R_{\mathrm{TX}}\right)$ can be evaluated as: $P_{d} \simeq \frac{\pi R_{\mathrm{TX}}^{2}}{\mathcal{A}}$. By substituting this in Equation (6), the optimal NAN coverage $R_{\mathrm{TX}}^{\mathrm{opt}}$, i.e., the maximum value of the transmission range that satisfies the collision constraint $\mathcal{T}_{C}$, is obtained by solving Equation (6) with respect to $R_{\mathrm{TX}}$ and by taking the maximum value:

$$
R_{\mathrm{TX}}^{\mathrm{opt}}=\sqrt{\frac{\mathcal{A}}{\pi P_{\mathrm{a}}}} \sqrt{1-\sqrt[G]{\frac{\frac{G K(K-1)}{2} \sqrt{\left(1-\mathcal{T}_{\mathrm{C}}\right)}-P_{\mathrm{in}}}{P_{\mathrm{a}}}}}
$$

From Equation (9) it results that the optimal coverage increases as the SG traffic demand $P_{\mathrm{a}}$ decreases, as expected.

In Table 1 we summarized the notation used for the parameters involved in the analysis along with their meaning, to improve the clarity of the manuscript.

Table 1. Adopted notation.

\begin{tabular}{ll}
\hline Symbol & Definition \\
\hline$K$ & number of NANs deployed in the network region \\
$\mathcal{A}$ & Area of the network region \\
$H_{\ell}^{i}$ & The $\ell$-th HAN gateway belonging to the $i$-th NAN \\
$H_{m}^{j}$ & The $m$-th HAN gateway belonging to the $j$-th NAN \\
$P_{C}^{i j}$ & Collision probability of the transmissions of NANs $i$ and $j$ \\
$P_{\mathrm{C}}^{\mathcal{O}}$ & Overall collision probability \\
$P_{\mathrm{a}}$ & Probability of an arbitrary HAN gateway belonging to the $i$-th NAN being active \\
$P_{\mathrm{in}}$ & Probability of an arbitrary HAN gateway belonging to the $i$-th NAN being inactive \\
$G$ & Number of HAN gateways \\
$R_{\mathrm{TX}}$ & Transmission range of an HAN gateway \\
$P_{d}$ & Probability of the distance $d(t)$ between two arbitrary HAN gateways belonging \\
$\mathcal{T}_{\mathrm{C}}$ & to two different NANs being smaller than $R_{\mathrm{TX}}$ \\
$R_{\mathrm{TX}}^{\mathrm{opt}}$ & Collision constraint, $i . e .$, the largest value of the overall collision probability tolerated by the SG scenario \\
$K_{\mathrm{opt}}$ & Optimal NAN coverage, $i . e .$, the maximum value of $R_{\mathrm{TX}}$ satisfying the collision constraint $\mathcal{T}_{\mathrm{C}}$ \\
\hline & The largest value of $K$ satisfying the collision constraint $\mathcal{T}_{\mathrm{C}}$ \\
\hline
\end{tabular}

\section{Numerical Results}

Here, we validate the theoretical results through Monte Carlo simulations. We consider $K=3$ NANs placed randomly in a squared region of area $\mathcal{A}$. Figure 2 shows the overall collision probability $P_{\mathrm{C}}^{\mathcal{O}}$ as a function of the SG traffic demand $P_{\mathrm{a}}$, for three different values of the normalized transmission range, i.e., $R_{\mathrm{TX}} / \sqrt{A}=\{0.01,0.02,0.03\}$. First, we note that the theoretical results match very well the simulation results. Then, when $P_{\mathrm{a}}$ decreases, $P_{\mathrm{C}}^{\mathcal{O}}$ decreases as well, since the probability of an HAN gateway being active decreases. Moreover, when $R_{\mathrm{TX}} / \sqrt{A}$ increases $P_{\mathrm{C}}^{\mathcal{O}}$ increases, since it is more likely that two HAN gateways are within a distance smaller than their transmission range. In the same figure, we also plot the optimal transmission range $R_{\mathrm{TX}}^{\mathrm{opt}} / \sqrt{A}$ given in Equation (9) as function of $P_{\mathrm{a}}$ for a value of the collision threshold equal to $\mathcal{T}_{\mathrm{C}}=0.05$. By comparison with the previous plot, we note that the theoretical results are once again confirmed. In fact, only if $R_{\mathrm{TX}} \leq R_{\mathrm{TX}}^{\mathrm{opt}}$, the collision constraint is satisfied. Figure 3 shows $P_{\mathrm{C}}^{\mathcal{O}}$ as a function of $P_{\mathrm{a}}$, for three different values of the number $G$ of HAN gateways, i.e., $G=\{10,20,30\}$. Again, the theoretical results match very well the simulation results. Moreover, when $G$ increases, $P_{\mathrm{C}}^{\mathcal{O}}$ increases as well. This result is reasonable, since it is more likely to find active HAN gateways. 

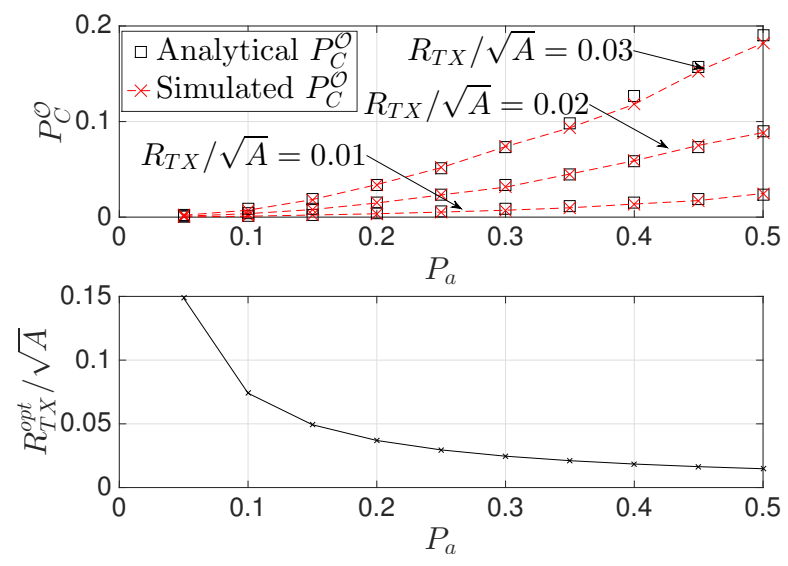

Figure 2. $P_{\mathrm{C}}^{\mathcal{O}}$ versus $P_{\mathrm{a}}$, for different values of $R_{\mathrm{TX}} / \sqrt{A}$.

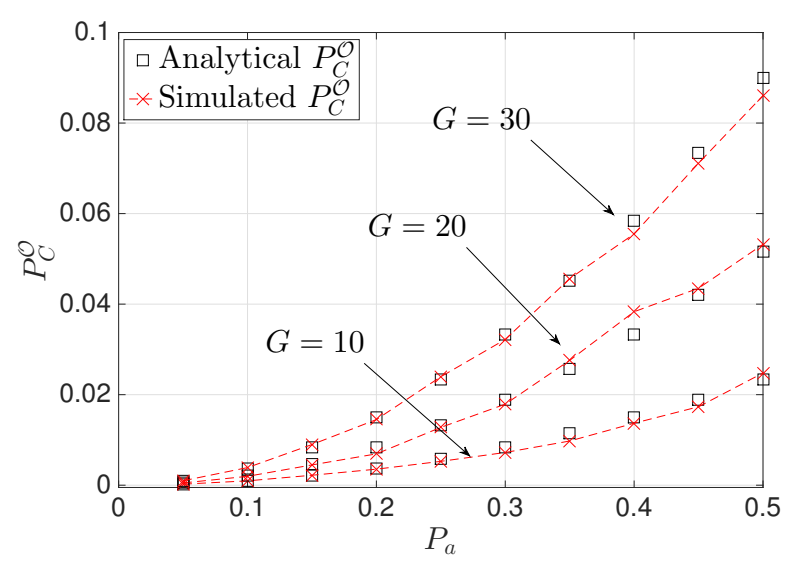

Figure 3. $P_{\mathrm{C}}^{\mathcal{O}}$ versus $P_{\mathrm{a}}$, for different values of $G$.

\section{Conclusions}

We developed a theoretical framework for the optimal deployment of multiple closely-located NANs over TVWS spectrum. Specifically, we derived through closed-form expressions the optimal values for both the number of NANs and their coverage, i.e., the maximum values assuring the given collision constraint. Such closed-form expressions reveled the highly non-linear relationship among the involved parameters as the number of NANs, their traffic demand, the number of HAN gateways and their transmission ranges. The developed analysis is crucial and preliminary for designing any effective coexistence protocol. Such an issue will be addressed in a future work along with optimization strategies for the DAU deployment.

Acknowledgments: This work has been partially supported by the National PON grants Databanc Chis and Fersat and the regional POR grant myOpenGov.

Author Contributions: The authors equally contributed to this work.

Conflicts of Interest: The authors declare no conflict of interest.

\section{References}

1. Khan, A.A.; Rehmani, M.H.; Reisslein, M. Cognitive Radio for Smart Grids: Survey of Architectures, Spectrum Sensing Mechanisms, and Networking Protocols. IEEE Commun. Surv. Tutor. 2015, 18, 860-898.

2. Zeng, F.; Xu, J. Leasing-Based Performance Analysis in Energy Harvesting Cognitive Radio Networks. Sensors 2016, 16, doi:10.3390/s16030305.

3. Zubair, S.; Syed Yusoff, S.K.; Fisal, N. Mobility-Enhanced Reliable Geographical Forwarding in Cognitive Radio Sensor Networks. Sensors 2016, 16, doi:10.3390/s16020172. 
4. Cacciapuoti, A.S.; Caleffi, M.; Paura, L. Widely Linear Cooperative Spectrum Sensing for Cognitive Radio Networks. In Proceedings of the 2010 IEEE Global Telecommunications Conference (GLOBECOM 2010), Miami, FL, USA, 6-10 December 2010; pp. 1-5.

5. Yu, R.; Zhang, Y.; Gjessing, S.; Yuen, C.; Xie, S.; Guizani, M. Cognitive Radio based Hierarchical Communications Infrastructure for Smart Grid. IEEE Netw. 2011, 25, 6-14.

6. Ul Hassan, N.; Yuen, C.; Atique, M.B. Tradeoff in Delay, Cost, and Quality in Data Transmission over TV White Spaces. In Proceedings of the IEEE ICC, Kuala Lumpur, Malaysia, 23-27 May 2016.

7. FCC. ET Docket 10-174: Second Memorandum Opinion and Order in the Matter of Unlicensed Operation in the TV Broadcast Bands; FCC: Washington, DC, USA, 2012.

8. Caleffi, M.; Cacciapuoti, A.S. Database access strategy for TV White Space cognitive radio networks. In Proceedings of the 2014 Eleventh Annual IEEE International Conference on Sensing, Communication, and Networking Workshops (SECON Workshops), Singapore, 30 June-3 July 2014; pp. 34-38.

9. Cacciapuoti, A.S.; Caleffi, M.; Paura, L. Optimal Strategy Design for Enabling the Coexistence of Heterogeneous Networks in TV White Space. IEEE Trans. Veh. Technol. 2016, doi:10.1109/ TVT.2015.2481007.

10. Cacciapuoti, A.S.; Caleffi, M. Interference analysis for secondary coexistence in TV white space. IEEE Commun. Lett. 2015, 19, 383-386.

11. Tushar, W.; Yuen, C.; Chai, B.; Huang, S.; Wood, K.L.; Kerk, S.G.; Yang, Z. Smart Grid Testbed for Demand Focused Energy Management in End User Environments. IEEE Wirel. Commun. Mag. 2016, arXiv:1603.06756.

12. Kuzlu, M.; Pipattanasomporn, M.; Rahman, S. Communication network requirements for major smart grid applications in HAN, NAN and WAN. Comput. Netw. 2014, 67, 74-88.

13. Harrison, K.; Sahai, A. Allowing sensing as a supplement: An approach to the weakly-localized whitespace device problem. In Proceedings of the IEEE DYSPAN, McLean, VA, USA, 1-4 April 2014; pp. 113-124.

14. Cacciapuoti, A.S.; Caleffi, M.; Marino, F.; Paura, L. Enabling Smart Grid via TV White Space Cognitive Radio. In Proceedings of the 2015 IEEE International Conference on Communication Workshop (ICCW), London, UK, 8-12 Jun 2015.

(C) 2016 by the authors; licensee MDPI, Basel, Switzerland. This article is an open access article distributed under the terms and conditions of the Creative Commons Attribution (CC-BY) license (http://creativecommons.org/licenses/by/4.0/). 